Historic, Archive Document

Do not assume content reflects current scientific knowledge, policies, or practices. 



\section{STOCK AND PRICE IIST}

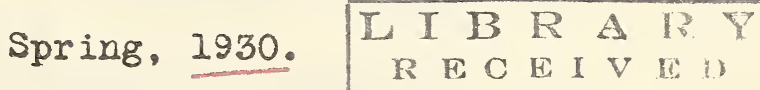

$$
\begin{aligned}
& \text { STABLER NURSERY * OCT } 131961 \text { \% }
\end{aligned}
$$

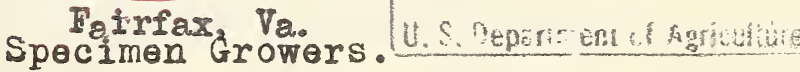

DECIDUOUS SHADE TREES

Each

Beech, American; Fagus ferruginea. Birch, White; Betula alba. $6 \mathrm{ft.} \quad \$ 2.75$

8 ft. 2.00

Dogwood, White Flowering; Cornus florida

$$
\begin{array}{lr}
6 \text { ft. } & 3.00 \\
5 \text { ft. } & 2.50 \\
4 \text { ft. } & 2.00 \\
3 \text { ft. } & 1.00 \\
2 \text { ft. } & .50
\end{array}
$$

Dogwood, Red Flowering; C. f. rubra.

$\begin{array}{rr}4 \text { ft. } & 5.00 \\ 3 \text { ft. } & 4.50 \\ 2 \text { ft. } & 2.50 \\ 18 \text { in. } & 1.50\end{array}$

Elm, American; Ulmus americana.

$\begin{array}{rr}10 \mathrm{ft.} & 2.50 \\ 8 \mathrm{ft.} & 2.00 \\ 6 \mathrm{ft.} & 1.50\end{array}$

Elm, Chinese; Ulmus pumila.
6 ft.
5 ft.
1.50
1.25

Gum, Sweet; Iiquidambar styraciflua. 
Gum, Sweet; (Continued)

\section{Each}

$$
\begin{aligned}
& 8 \text { ft. } \quad \$ 3.00 \\
& 6 \mathrm{ft} \text {. } \quad 2.00
\end{aligned}
$$

Horse-Chestnut; Aeschulus hippocastanum.

$8 \mathrm{ft}$. $\quad 3.00$ Linden, American; Tilia americana.

Maple, Japanese Redieaf.

$$
\begin{array}{rr}
12 \mathrm{ft.} & 3.00 \\
10 \mathrm{ft} . & 2.50 \\
8 \mathrm{ft.} & 2.00 \\
6 \mathrm{ft} . & 1.50
\end{array}
$$

(See under Dociduous Shrubs) Maple, Red or Scarlet; Acer rubrum.

$$
\begin{aligned}
12 \mathrm{ft} . & 3.00 \\
10 \mathrm{ft.} & 2.50 \\
8 \mathrm{ft} . & 2.00
\end{aligned}
$$

Maple, Norway; A. platanoides.

$$
\begin{array}{rrr}
10 & \mathrm{ft} . & 2.50 \\
8 \mathrm{ft} . & 2.00 \\
6 \mathrm{ft.} & 1.50
\end{array}
$$

Maple, Schwedler's; A. p. Schwedleri.

Maple, Sugar; A. saccarum.

$$
4 \mathrm{ft} \text {. } \quad 2.00
$$

Oak, Pin: Quercus palustris.

$$
6 \mathrm{ft.} \quad 1.50
$$

$\begin{array}{ll}8 \mathrm{ft} . & 2.50 \\ 6 \mathrm{ft} & 2.00\end{array}$

Oak, Red; Q. rubra.

$$
\begin{array}{rrr}
12 & \text { ft. } & 4.00 \\
10 \mathrm{ft.} & 3.50 \\
6 \mathrm{ft.} & 2.00
\end{array}
$$

Oak, Scarlet; 2. coccinea.
$8 \mathrm{ft}$.
6 ft.
2.50

Oak, White; Q. alba.

$\begin{array}{lll}6 & \text { ft. } & 2.00 \\ 5 & \text { ft. } & 1.50\end{array}$


Oak, Willow; Quercus phellos.

Each

6 ft.

$\$ 2.00$

Plane, 5 ft. 1.50

a.

$8 \mathrm{ft}$.

2.50

Plane, (Sycamore); Platanus occidentalis.

$8 \mathrm{ft}$.

2. 50

Poplar, Lombardy; Populus nigra italica.

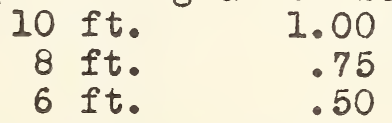

Walnut, Stabler Black; Juglans nigra.
$6 \mathrm{ft}$.
6.00
$5 \mathrm{ft}$.
5.00
$4 \frac{1}{2}$ t.
$4 \mathrm{ft}$.
4.00
3.00

Willow, Solomons; Salix salamonii.
One jear plants,
$6 \mathrm{ft}$.
.50
$4 \mathrm{ft}$.
.30

\section{EVERGREENS.}

In comparing the prices of various growers, we urge that buyers of Evergreens investigate as to quality also. The specimens offered here a.I have compact symmetrical tops, of the best type for the variety, and have been transplanted, in the nursery, at least once in the past two years. Each carries with it an excellent ball of earth on the roots, which makes it heavier to move, but helps greatly to insure success to the planter. 
CONIFEROUS EVERGREENS

Each

Arborvitae, American; Thuja occidentalis.

$$
\begin{array}{ll}
7 \text { ft. } & \$ 7.00 \\
6 \text { ft. } & 6.00 \\
5 \text { ft. } & 5.00 \\
4 \text { ft. } & 3.75 \\
3 \frac{1}{2} \text { ft. } & 3.25 \\
2 \text { ft. } & 1.50
\end{array}
$$

Arborvitae, Erect; T. o. pyramidalis.

$$
\begin{array}{ll}
6 \text { ft. } & 6.50 \\
5 \text { ft. } & 5.00 \\
4 \text { ft. } & 4.00 \\
3 \text { ft. } & 3.00
\end{array}
$$

Arborvitae, Compact; T. o. compacta.

$$
\begin{array}{ll}
3 \text { ft. } & 4.00 \\
2 \frac{1}{2} \text { ft. } & 3.50 \\
2 \text { ft. } & 2.50 \\
1 \frac{1}{2} \text { ft. } & 1.50
\end{array}
$$

Arborvitae, Tom Thumb; T. o. ellwangeriana.
$2 \mathrm{ft}$.
2.00

Arhorvitae, Chinese; T. orientalis.

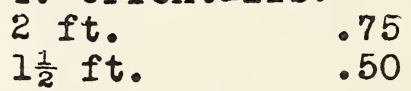

Arborvitae, Chinese Cornpact; T. or. var.

$$
\begin{array}{lr}
4 \mathrm{ft.} & 10.00 \\
2 \frac{1}{2} \mathrm{ft.} & 3.50 \\
2 \mathrm{ft.} & 2.50
\end{array}
$$

Cedar, Red; (See under Junipers).

Cypress, Japanese (Called Retinosporas) Blue C.; Chamaecyparia squarrosa

$$
\begin{array}{lr}
6 \text { ft. } & 10.00 \\
5 \text { ft. } & 7.50 \\
4 \text { ft. } & 5.00 \\
3 \text { ft. } & 3.75 \\
2 \text { ft. } & 2.00
\end{array}
$$


CONIFEROUS EVERGREENS (Continued)

Each

Cypress, Japaneso;(Called Retinosporas). Plumed Cypress; C. pisifera plumosa.
7 ft.
6 ft.
$5 \mathrm{ft.}$
4 ft.
3 ft.
2 ft.

$\$ 10.00$

7.50

0.00

Golden Plumed C.; C. p. p. aurea. 2.00

$$
\begin{array}{lll}
3 \frac{1}{2} \text { ft. } & 5.00 \\
3 & \text { ft. } & 4.50 \\
2 \frac{1}{2} \text { ft. } & 3.50 \\
2 & \text { ft. } & 2.00
\end{array}
$$

Golden Dwarf C.; C. p. p. a. nana.

$$
\begin{aligned}
& \text { I量 ft. } \\
& 1 \text { ft. } \quad 1.75
\end{aligned}
$$

Sawara C.; Chamaecyparis pisifera.

$$
\begin{aligned}
& 2 \frac{1}{2} \mathrm{ft} \text {. } \\
& 2 \text { ft. } \quad 1.75
\end{aligned}
$$

Golden Sawara C.; C. p. aurea.

$$
\begin{array}{ll}
6 \text { ft. } & 7.50 \\
5 \text { ft. } & 6.25 \\
4 \text { ft. } & 5.00 \\
3 \text { ft. } & 3.50 \\
2 \text { ft. } & 2.00
\end{array}
$$

Threadbranched C; C. p. filifera. $1 \frac{1}{2} \mathrm{ft}$. $\quad 3.00$ Fir, Fraser's Balsam;.Abies fraseri

$$
\begin{array}{ll}
3 \mathrm{ft} . & 4.00 \\
2 \mathrm{ft} & 3.00
\end{array}
$$

Hemlock; Tsuga canadensis.

$$
\begin{array}{ll}
5 \mathrm{ft.} & 6.25 \\
4 \mathrm{ft.} & 5.00 \\
3 \mathrm{ft.} & 4.00
\end{array}
$$


CONIFEROUS EVERGREENS (Continued)

Each

Hemlock (Continued).

$$
\begin{array}{lll}
2 \frac{1}{2} \text { ft. } & 3.50 \\
2 & \text { ft. } & 2.00 \\
1 \frac{1}{2} \text { ft. } & 1.50
\end{array}
$$

Juniper, Canadian; Juniperus canadensis.

$$
\begin{aligned}
& \text { Width } 5 \text { ft. } 10.00 \\
& 4 \text { ft. } \quad 7.50 \\
& 3 \frac{1}{2} \text { ft. } \quad 5.00 \\
& 3 \text { ft. } \quad 3.50 \\
& 2 \text { ft. } 2.00
\end{aligned}
$$

Juniper (Redcedar); J. virginiana.

$\begin{array}{lll}8 & \text { ft. } & 8.00 \\ 7 & \text { ft. } & 7.00 \\ 6 & \text { ft. } & 5.50 \\ 5 & \text { ft. } & 3.75 \\ 4 & \text { ft. } & 3.00 \\ 3 & \text { ft. } & 1.75 \\ 2 & \text { ft. } & 1.00\end{array}$

Juniper, Pfitzer's; J. Chinensis ver.

Width 3 ft. $\quad 4.50$

$2 \frac{1}{2}$ ft. $\quad 3.75$

2 ft. $\quad 3.00$

l表 ft. 2.25

Juniper, Savin; J. sabina.

$\begin{array}{lll}\text { Width } & 3 \text { ft. } & 4.50 \\ & 2 \frac{1}{2} \mathrm{ft} . & 3.75 \\ 2 \mathrm{ft.} & 3.00 \\ & 1 \mathrm{ft} . & 2.00\end{array}$

Juniper, European; J. communis.

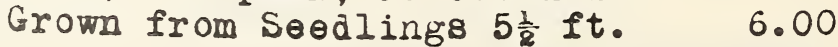

$\begin{array}{lll}5 & \text { ft. } & 5.00 \\ 4 & \text { ft. } & 4.00\end{array}$

Juniper, Blue; J. communis var.

Grown from Cuttings $2 \frac{1}{2} \mathrm{ft}$.

2.50

2 ft.

2.00

1 ft.

.75 
CONIFEROUS IVERGREENS (Continued)

Each

Pine, Austrian; Pinus nigra.

$1 \frac{1}{2}$ ft.

1 ft.

$\$ 1.50$

.75

Pine, Loblolly; Pinus taeda.

2.50

5 ft.

4 ft.

2.00

3 ft.

1.50

Pine, Scotch; Pinus sylvestris.

$\begin{array}{ll}2 \text { ft. } & 1.75 \\ \text { lit. ft. } & 1.00\end{array}$

Pine, White; Pinus strobus.

$\begin{array}{lll}6 & \text { ft. } & 6.00 \\ 5 & \text { ft. } & 4.00 \\ 4 & \text { ft. } & 3.00 \\ 3 & \text { ft. } & 2.00 \\ 2 & \text { ft. } & 1.00\end{array}$

Spruce, Coloradogreen; Picer pungens.

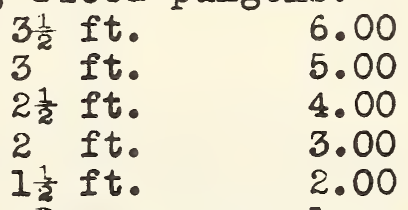

Spruce, Colorado Blue; $P$. pungens glauca.

$2 \frac{1}{2} \pm t . \quad 7.50$

2 ft. $\quad 5.50$

$1 \frac{1}{2}$ ft. $\quad 2.25$

Spruce, Koster's Blue; P. p. kosteriana.
$5 \frac{1}{2} 1 t$.
$4 \frac{1}{2} 1$ t.
50.00
30.00

Spruce, Douglass'; Pseudotsuga taxifolia.

$1 \frac{1}{2} \mathrm{ft}$. 1.00

Spruce, White; Picea canadensis.
2 ft.
$1 \frac{1}{2}$ ft.
1.50 
BROAD IEAVED EVERGREENS

Each

Arbutus Bush; Abelia gxandiflora.

$$
\begin{array}{ll}
1 \frac{1}{2} & f t . \\
1 & f t .
\end{array}
$$

$\$ 0.75$

.50

Andromeda: Loucothoe catesbari.

$$
\begin{aligned}
& 1 \frac{1}{2} \text { ft. } \\
& 1 \text { ft. } \\
& 1.75 \\
& 1.50
\end{aligned}
$$

Box, Dwarf or Bush; Buxus sempervirens suffruticosa.

$$
\begin{array}{rr}
10 \text { in. } & 1.00 \\
6 \text { in. } & .45 \\
4 \text { in. } & .25 \\
\text { 2-yr. plants, not balied, } & .15
\end{array}
$$

Box, Common or Tree; B. sempervirens.

$$
\begin{array}{ll}
2 & \text { ft. } \\
1 \frac{1}{2} & \text { ft. } \\
15 & \text { in. } \\
12 & \text { in. } \\
10 & \text { in. } \\
6 & \text { in. }
\end{array}
$$$$
1.00
$$

2-yr. plants, not ballod.

Plerse Note:-

Ungrafted Hollies vary greatly in berry boaring habits and in boauty of follage -.. in fact, our tests hare shown that less than one seodling in each hundred will be of superior ornamental value and hardiness.

Therefore we have propagated, and now offer our customers, two grafted varietios of outstanding merit. But staminate trees that will never bear borries can be furnishod on special quotation if requested. 
BROAD LEAVED EVERGREENS

Each

Holly, Collier; Ilex opaca variety.

$\begin{array}{lll}4 & \text { ft. } & \$ 8.00 \\ 2 \frac{1}{2} & \text { ft. } & 5.00 \\ 2 & \text { ft. } & 4.00 \\ 1 \frac{1}{2} & \text { ft. } & 3.00 \\ 1 & \text { ft. } & 1.50\end{array}$

Holly, Wooster; Ilex opaca var.

$\begin{array}{lll}3 \text { ft. } & 6.00 \\ 2 & \text { ft. } & 4.00 \\ 1 \frac{1}{2} \text { ft. } & 3.00 \\ 1 & \text { ft. } & 1.50\end{array}$

Magnolia grandiflora.

$\begin{array}{lll}5 & \text { ft. } & 5.00 \\ 4 & \text { ft. } & 4.00 \\ 2 & \text { ft. } & 2.00\end{array}$

Mountain Laurel; Kalmia latifolia.

$\begin{array}{llr}4 & \text { ft. } & 4.50 \\ 3 & \text { ft. } & 3.50 \\ 2 & \text { ft. } & 2.50 \\ 1 & \text { ft. } & .75\end{array}$

Privet, Waxleaf; Ligustrum lucidum.

$$
\begin{array}{lll}
2 & \text { ft. } & 2.00 \\
1 \frac{1}{2} & \text { ft. } & 1.25
\end{array}
$$

Rosebay; Rhododendr on maximum.

Plosso Noto:-

Rhododendron, Iaurel, and Andromeda must bo plantod in an acid soil. preferably in a situation shaded from tho morning sun

$\begin{array}{lll}4 & \text { ft. } & 4.00 \\ 3 & \text { ft. } & 3.00 \\ 2 \frac{1}{2} \text { ft. } & 2.50 \\ 2 & \text { ft. } & 2.00 \\ 1 \frac{1}{3} \text { ft. } & 1.50\end{array}$




\section{DECIDUOUS SHRUBS}

Each

Acacia, Rose; Robinia hispida.

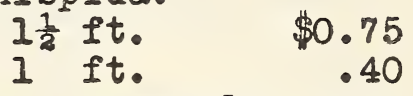

Almond, Double Flowering; Amygdalus nana.
3 ft.
.75
$2 \frac{1}{2}$ ft.
.50
2 ft.
.40

Barberry, Japanese; Berberis thunbergi.

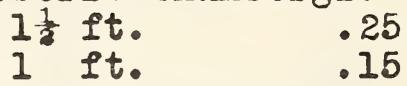

(For quantity prices see Hedge Plants)

Butterfly Bush; Buddleia davidi.

$$
3 \text { ft. }
$$

Crape Myrtle; Lagerstroemia indica.

Please Note:-

Crape Myrtlo does not become

hardy for this climate until it is

about four years old. Requires

winter protection, and should not

be planted in soil that is ever

too wet.

Pink, White, and Purple varieties:

$$
\text { Two years old, } 2 \text { ft. } \quad 1.00
$$

Red variety,

Two years old, 2 ft. $\quad 1.25$

Callicarpa purpurea.

Clethra alnifolia.

2 ft. $\quad .50$

2 fft. $\quad .50$

I $\frac{1}{2}$ ft. $\quad .40$

Dogwood, Flowering; Cornus florida.

(See under Trees, Page 1.) 
DECIDUOUS SHRUBS (Continued)

Each

Dogwood; Red Osier; Cornus alba.

$$
\begin{array}{lll}
2 & \text { ft. } & \$ 0.25 \\
1 \frac{1}{2} \text { ft. } & .20
\end{array}
$$

Forsythia, Golden Bell; Forsythia fortunei.

2 ft. .35

Fringe Tree; Chionanthus virginica.

2 ft. .85

Hydrangea, Snowhill; H. arborescens.

$$
\begin{array}{lll}
\text { Clumps, } & 1 \frac{1}{2} \mathrm{ft} . & .75 \\
\text { Plants, } & \text { l } \frac{1}{2} \mathrm{ft} . & .40
\end{array}
$$

Hydrangea, Fall Blooming; Hyarangea paniculata grandiflora.

$$
\begin{array}{lll}
3 & \text { ft. } & 1.00 \\
2 & \text { ft. } & .75 \\
1 \frac{1}{2} & \text { ft. } & .30
\end{array}
$$

Maple, Japanese Bloodleaf; Acer palmatum atropurpureum. Grafted specimens,

$$
\begin{array}{lll}
1 \frac{1}{2} & \text { ft. } & 5.00 \\
15 & \text { in. } & 3.50 \\
1 & \text { ft. } & 2.50
\end{array}
$$

Quince, Jepanese; Chaenoneles japonica.

$$
\begin{aligned}
& 2 \text { 1t. } .60
\end{aligned}
$$

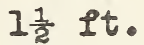

Spiraea, Dwarf Pink; S. Anthony Waterer. Clumps, $1 \frac{1}{2}$ ft. .50 Spiraea, Thunberg's; S. thuubergi. Spiraea vanhouttei.

$1 \frac{1}{2}$ ft. .50

$2 \mathrm{ft.} \quad .50$

Weigela, Pink; Weigela rosea.

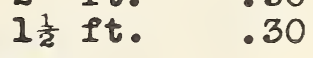

Weigela Eva Rathke.

$$
2 \text { ft. } \quad .75
$$$$
2 \text { It. } .80
$$ 
EVERBIOOMING BUSH ROSES

Each

CHARLES K. DOUGIAS -- A Vigorous, large-flowered, semi-double

Hybrid Tea. Flaming scarlot. \$0.90

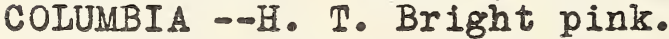

subject to suaburn if planted in dry soil.

DOUBIE WHITE RIIIARNEY -- White;

very pleasant fragrance. H. T. 1.00 DUCHESS OR WEIIINGTON $-\mathrm{H}$. T.

A vigorous and productive bush,

that bears large yellow buds,

opening into semi-double blooms.1.00 ECARIATE - - H. T. A hardy bush

that bears numerous scarlet

flowers constantly. For garden

decoration, not for cut flowers. .90 FRANCIS SCOTT KEY -- H. T. Brilliant

crimson, very large, double. 1.00

FRAU KARI DRUSCHKI -- Hybrid Per-

petual. Large, snow-white blooms .75

GRUSS AN TEPIITZ -- A very vigorous

and productive crimson Hybrid Tea .60 KILLARNEY - - H. T. Bright Pink,

with tall stiff thorny stoms.

GRUSS AN AACHEN -- Polyantho.

Blush pink, with very short stems.

A good dware bedding variety. 1.00

KAISERINE AUGUSTA VICTORIA - - H. T.

Excellent ivory-white variety. 1.00

OPHELIA - - H. T. Shapely blush

buids borne on long. stiff,

smooth stems. One of the best

for cut flowers.

1.00 
EVIRBLOOMING BUSH ROSES (Continued)

Erch

RADIANCE - - H. T. Large, double,

tulip-like flowers of bright

pinix. Fragrant. More largely

planted, and more generally suc-

cessful, than any other variety. \$0.90

RED RADIANCE -- H. T. Differs

from Radiance in color only.

Large flowers of good lasting

red color are produced freely

from Hay until frost.

HARDY CIIMBING ROSES

Each

AMERICAN PILLAR -- Pink, with white center.

DR. VAN FLEET -- Flosh pink. $\$ 0.75$ EXCELSA - Crimson, blooms late. .40 MARY WALIACE -- Pink, pillar rose. .75 PAUL'S SCARLET -- Flaming scarlot. 75 SIIVER MOON -- White, yellow heart .75 ZEPHIRINE DROUHIN -- Bright pink. .60

\section{SHRUB ROSES}

Each

HARISON'S YELIOW -- Double yellow. .75 HUGONIS -- Single yellow, early. .75 S. J. GROOTENDORST -- Frilled red, nearly everblooming. 


\section{HEDGE PIANTS}

Please Note:- The dimensions of Hedge Plants are given by both height, and the spacing recommended for singleline planting under most conditions. To compute the number of plants needed for any particular hedge, find out the length of the proposed hedge in inches, and then divide by the spacing of the plant to be used. Thus, if a hedge is desired of Barberry, to be eighteen inches high when planted, and fifty feet long, then forty 18-inch Barberry are nooded, if spaced 15 inches.

DECIDUOUS HEDGE PLANTS

Height Space Per Per in. in. 10. 100.

Barberry,

Japanese,

$\begin{array}{llrr}12 & 12 & \$ 1.50 & \$ 10.00 \\ 18 & 15 & 2.50 & 15.00\end{array}$

Privet,

$\begin{array}{rrrrr}\text { California, } & 12 & 6 & .50 & 4.00 \\ & 18 & 8 & .75 & 5.00 \\ & 24 & 10 & 1.00 & 7.50\end{array}$

EVERGREEN HEDGE PLANTS

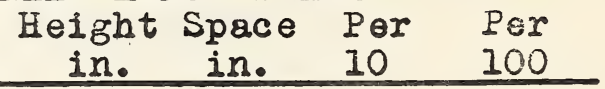

Arborvitae,

American, B. \& B.

$12 \quad \begin{array}{llll}18 & 6.00 & 50.00\end{array}$

Fir, Fraser's, B. \& B.

$\begin{array}{lll}12 & 18 & 14.00 \\ 18 & 18 & 20.00\end{array}$


EVERGREEN HEDGE PLANTS (Continued) \begin{tabular}{ccc} 
Height Space Per & Per \\
in. In. & 10. & 100. \\
\hline
\end{tabular}

Hemlock,

Canadian, B.\& B.I2

\section{8}

$18 \$ 12.00 \$ 100.00$

$\begin{array}{llll}24 & 24 & 30.00 & 225.00\end{array}$

Box, Dwarf, ballea.

$\begin{array}{llll}36 & 30 & 45.00 & 350.00\end{array}$

Box, Tree, balled

$\begin{array}{llll}3 & 4 & 2.00 & 12.00 \\ 4 & 4 & 4.00 & 35.00\end{array}$

$\begin{array}{lrrrr} & 4 & 6 & 2.00 & 12.00 \\ \text { B.\& B. } & 6 & 8 & 4.00 & 35.00 \\ & 8 & 8 & 5.00 & 40.00 \\ \text { Waxleaf. } 12 & 10 & 8.00 & 70.00\end{array}$

EVERGREIN SCREEN PIANTS Height Space Per

$\frac{\text { ft. ft. } 10}{\text { Juniper, European Upright, B.\& B. }}$

$$
\begin{array}{lll}
1 & 1 & \$ 5.00 \\
1 \frac{1}{2} & 1 & 10.00 \\
2 & 1 \frac{1}{2} & 15.00 \\
2 \frac{1}{2} & 2 & 20.00
\end{array}
$$

Pine, Loblolly, B. \& B.

$$
\begin{array}{lll}
3 & 6 & 15.00 \\
4 & 6 & 20.00 \\
5 & 6 & 25.00
\end{array}
$$

Pine, Scotch, B. \& B.

Pine, 俰ite, B. \&. B.

$$
\begin{array}{llr}
1 & 2 & 5.00 \\
1 \frac{1}{2} & 3 & 10.00 \\
2 & 3 & 17.50
\end{array}
$$

$$
\begin{array}{lll}
3 & 6 & 20.00 \\
4 & 6 & 30.00
\end{array}
$$


EVERGREEN SCREEN PIANTS (Continued) Height Space Por Por

ft. ft. 10. 100.

Spruce, Norway, B. \& B.

$\begin{array}{lllr}1 & 2 & \$ 5.00 & \$ 45.00 \\ 1 \frac{1}{2} & 3 & 10.00 & 90.00 \\ 2 & 3 & 15.00 & 125.00 \\ 3 & 4 & 25.00 & 200.00 \\ 4 & 4 & 35.00 & \end{array}$

Note:- Norway Spruce makes an excellent Christmas Tree. We can, in season, supply them in any quantity, cut, at about half the above rates. Get our prices, whether you can use one or a carload.

Redcedar, B. \& B.

$$
\begin{array}{llrl}
1 & 1 & 5.00 & 40.00 \\
1 \frac{1}{2} & 1 & 7.50 & 60.00 \\
2 & 1 \frac{1}{2} & 10.00 & 90.00
\end{array}
$$

\section{IIVING CHRISTMAS TREES}

Each

Please Note:-

It is a splendid idea to here the Christmas Tree planted permanently on the lawn. But only nursery-grown evergreens of "Specimen" quality should be so used. We are prepared to give special service in case larger trees are desired than those quoted in the lists above. Normay Spruce, B. \& B. $4 \mathrm{ft}$ Colorado spruce, B. \& B. $4 \mathrm{ft}$. Bedcedar, B. \& B. 8 ft. 